\title{
3 Research Square \\ Dynamics and Transcriptome Analysis of Starch and Sucrose Metabolism During Corm Development in Freesia Hybrida
}

\section{Li Ma}

Shanghai Jiao Tong University

\section{Suqin Ding}

Shanghai Jiao Tong University

Zi Yan

Shanghai Jiao Tong University

Dongqin Tang ( $\nabla$ dqtang@sjtu.edu.cn )

Shanghai Jiao Tong University https://orcid.org/0000-0002-5066-5065

\section{Research article}

Keywords: Freesia, corm formation and development, starch and sucrose metabolism, transcriptome, gene expression

Posted Date: May 21st, 2020

DOI: https://doi.org/10.21203/rs.3.rs-28444/v1

License: (c) (i) This work is licensed under a Creative Commons Attribution 4.0 International License. Read Full License 


\section{Abstract}

Background Starch and sucrose metabolism plays a crucial role in the formation and development of bulbs in bulbous plants, which, however, remains unclear and unexplored in the corms of Freesia hybrida, one kind of famous bulbous flower. Herein, we investigated the dynamics of the major form of carbohydrates and related enzyme activities and profiled the transcriptome of freesia corms at four developmental stages with the aim to reveal the relation of starch and sucrose metabolism to corm development and the transcriptional regulation of this metabolic process.

Results The content of starch, sucrose and soluble sugars followed an overall upward trend across the corm developmental stages. Activities of the adenosine diphosphoglucose pyrophosphorylase, starch branching enzyme and $\beta$-amylase generally followed the pattern of the starch and sucrose level. Activities of sucrose phosphate synthase increased from corm formation till the initial swelling stage and subsequently reached a plateau. Activities of invertase and sucrose synthase peaked at the later rapid swelling stage. Transcriptome analysis revealed a total of 100,999 unigenes, out of which 44,405 unigenes were annotated. Analysis based on Clusters of Orthologous Groups suggested that carbohydrate transport and metabolism (9.34\% of the sequences) was prominent across the corm developmental process. Totally 3427 differentially expressed genes were identified and the enrichment analysis directed starch and sucrose metabolism a critical pathway in corm development especially at the rapid swelling stage. Genes encoding key carbohydrate-metabolizing enzymes were identified and their differential expression related to corm development was explored.

Conclusions The comparative transcriptome analysis discloses essential roles of starch and sucrose metabolism and the genetic mechanism related to the corm development. The results construct a valuable resource pool for further molecular-level studies, which are helpful for metabolic regulation of carbohydrates and improvement in molecular breeding of Freesia hybrida.

\section{Introduction}

Freesia (Freesia spp.), a species of Iridaceae family and indigenous to South Africa [1], is one of the most popular cut flowers in the world for its pleasant flower aroma and diverse flower colors. Freesia is a bulbous herbaceous perennial and it sprouts in the autumn and blooms in winter to early spring. It was introduced from the native habitat into other countries and subject to hybridization breeding, producing assorted commercialized modern freesia (Freesia hybrida). Corms are the propagative organ, thus the quality of corms determines the healthiness of the plant and then the ornamental value of freesia. Previous studies suggest that development of storage roots was paralleled with complex physiological activities including carbohydrate, enzyme, and hormone dynamics [2-4]. A wealth of studies regarding this have been conducted on bulbous flowers such as lilies [5-7] and tulips [2,3, 8], whereas freesia remains unexplored according to our knowledge.

Starch is a major storage form of carbohydrate and an energy carrier in plant storage organs. Starch content was also proposed to be a key judge for lily bulb quality [9]. During lily bulblet formation, starch in mother bulb degraded in favor of daughter bulb development [7]; thereafter, an continuous accumulation of starch was observed till bulblet maturation. Apart from being a labile source of carbon and energy [10], sucrose is the major form of carbohydrate for long distance translocation and serves as a signaling [11] and osmotic regulating substance [12] as well as a substrate for starch production in many plants. Sucrose was reported to be the major form of soluble sugar accounting for cell division and enlargement during tulip stolon development [3]. Soluble carbohydrates, typically sucrose, were described to be strong triggers of potato tuberization [13]. A concomitant accumulation of starch and sucrose was observed across tube and bulblet development $[5,13]$.

Loading [MathJax]/jax/output/CommonHTML/jax.js 
Starch and sucrose metabolism is closely related to a series of key enzymes. Sucrose synthase (SuSy) catalyzes the reversible synthesis of sucrose, but hydrolytic activity is suggested to play a leading role [3]. Sucrose phosphate synthases (SPS) promote sucrose synthesis and invertases (INV), including neutral invertases (NI) and acid invertases (AI), are responsible for irreversible degradation of sucrose. SuSy and INV synergistically break down sucrose into fructose and glucose, which can subsequently join in starch synthesis pathways $[7,14]$. Adenosine diphosphoglucose pyrophosphorylase (APGase) initiates the synthesis of starch by catalyzing the formation of adenosine diphosphate glucose (ADPG), a precursor of starch synthesis [15]. Subsequently, soluble starch synthase and granule-bound starch synthase catalyze the starch synthesis using the substrate, ADPG. Starch branching enzymes (SBE) add branches to glucan to form amylopectin. Amylases (AMY) is a key enzyme that cleaves starch into soluble sugars to sustain plant growth. Miao et al. [2, 3] revealed that the development of Tulipa edulis stolons was accompanied with a sugar consumption and starch production via the action of enzymes. Vishnevetsky et al. [4] found that content of carbohydrates were strongly correlated to corresponding enzyme activities in Nerine sarniensis bulbs at different developmental stages. The significance of metabolic enzymes in carbohydrate metabolism in developmental bulbs was further revealed in oriental hybrid lily 'Sorbonne' [16] and Gladiolus hybridus [17].

Still, there is a lack of information in molecular mechanisms related to carbohydrate metabolism in freesia corm development. Genes related to enzymes involved in starch and sucrose metabolism remain unexplored. In view of this, in the current study, the dynamic changes in levels of starch and sucrose and key enzymes involved were monitored and transcriptomic analysis of carbohydrate metabolism was performed during freesia corm development, with the aim to bridge the knowledge gap in functions of major carbohydrates regulating freesia corm development, both physiologically and transcriptionally. The next-generation high-throughput sequencing method was employed in this study to sequence the transcriptome of freesia corms. This sequencing method applies not only to plants with known genome but also to those lack of reference genomic sequences, and has already been successfully attempted in diverse plants for transcriptomic profiling [18].

To our knowledge, this is the first thorough investigation of starch and sucrose metabolism across corm development in freesia at the physiological and transcriptional level, which is of special importance for quality control of corms and informative for development of proper cultivation and breeding practices of freesia.

\section{Results}

\section{Starch and sucrose dynamics}

\section{Starch metabolism and corresponding enzyme dynamics}

Description of four stages of corm development was displayed in Additional file 1: Tab. S1. Dynamics of starch content and the enzyme activities are shown in Fig. 1. Over the new corm developmental stages, starch content saw an overall increasing trend with the minimum (30.94 mg/g.FW) and maximum value $(65.04 \mathrm{mg} / \mathrm{g} . \mathrm{FW})$ appearing, respectively, at the beginning and the end of the sampling event. Starch content increased slowly within 110 days since mother corm embedding. Thereafter, the content rose rapidly reaching $\sim 60 \mathrm{mg} / \mathrm{g}$.FW from 110 to $130 \mathrm{~d}$ (transition from initial swelling stage to rapid swelling stage), followed by a slightly decrease downward ( 130-150 d) and subsequent increase at the corm maturation stage ( 150-190 d).

Beta-AMY activities were rather dynamic. Specifically, $\beta$-AMY activities increased from 273 to $408 \mu \mathrm{g} / \mathrm{min} / \mathrm{g}$.FW during the corm formation stage (60-90 d). After transient decrease, the enzyme activity increased sharply and peaked at 120 $\mathrm{d}$ (appearance of flower bud), reaching $498.33 \mu \mathrm{g} / \mathrm{min} / \mathrm{g}$.FW, which was followed by marked decrease in the next

Loading [MathJax]/jax/output/CommonHTML/jax.js ]uctuations during maturation stage. Throughout the corm lifecycle, an 
overall increasing trend of SBE activities was observed, especially at the rapid swelling stage. During the maturation stage (150-180 d), the increasing rate of enzyme activity was almost flat. The value of the highest enzymatic activity was $\sim 4$ fold that of the minimum at the beginning of sampling. Overall, APGase activities increased across the corm development stages, especially during the formation (60-90 d) and maturation stages (140-190 d), while fluctuations were observed during corm swelling (110-140 d). The minimum ( $80 \mu \mathrm{g} / \mathrm{min} / \mathrm{g} . \mathrm{FW})$ and maximum $(\sim$ $240 \mu \mathrm{g} / \mathrm{min} / \mathrm{g} . \mathrm{FW}$ ) values appeared at the first and last sampling event, respectively.

\section{Sucrose metabolism and corresponding enzyme dynamics}

Figure 2 depicts changes of sugar content and corresponding enzyme activities across new corm development. On average, levels of sucrose and soluble sugars followed an increasing trend across the corm developmental process. By comparison, the increasing trend was more pronounced for sucrose than for soluble sugars for which more fluctuations were observed. At the formation stage (60-90 d), sucrose and soluble sugars both kept at a relatively constant level and then increased rapidly at times during corm swelling. Even at the maturation stage, sucrose content rose rapidly to reach a maximum (4.43 mg/g.FW), which was 3.69 times of the lowest value (1.2 mg/g.FW) at the first sampling event. For soluble sugars, the maximum and minimum values were 3.14 and $11.06 \mathrm{mg} / \mathrm{g}$.FW, respectively.

SPS activities kept relatively constant $(\sim 350 \mu \mathrm{g} / \mathrm{min} / \mathrm{g}$.FW) during the formation stage (60-90 d), then increased sharply reaching maximum ( $640 \mu \mathrm{g} / \mathrm{min} / \mathrm{g}$.FW) during corm swelling (100-140 d) and subsequently kept relatively stable except a marked drop at $150 \mathrm{~d}$. Comparatively, the profile of SuSy activities exhibited a slightly different pattern. The enzymatic activity showed monotonous decline from 705 to $433 \mu \mathrm{g} / \mathrm{min} / \mathrm{g}$.FW during the formation stage, then rose rapidly arriving at maximum $(\sim 1006 \mu \mathrm{g} / \mathrm{min} / \mathrm{g}$.FW) during the swelling period and finally dropped back to the beginning level when corms began mature. NI activities increased continuously from $60 \mathrm{~d}(88.47 \mu \mathrm{g} / \mathrm{min} / \mathrm{g}$.FW) to $150 \mathrm{~d}$ $(\sim 180 \mu \mathrm{g} / \mathrm{min} / \mathrm{g} . \mathrm{FW})$ followed by a sustaining drop arriving at $126.19 \mu \mathrm{g} / \mathrm{min} / \mathrm{g} . \mathrm{FW}$ at the end. Averagely, Al activities followed an initial increasing and subsequent decreasing trend as corms grew except the presence of a drop at $100 \mathrm{~d}$. The maximum and minimum activities were 64.2 and $123.27 \mu \mathrm{g} / \mathrm{min} / \mathrm{g}$.FW, respectively.

\section{Correlations between starch and sucrose content, enzyme activities and corm size}

The correlation analysis shows that activities of SPS, AGPase and SBE were positively and significantly correlated with sucrose and corm diameter $(P<0.01)$, which all were significantly correlated to starch content (Table 1$)$. Besides, $\mathrm{NI}$ exhibited a positive and significant relationship with starch content and corm dimeter $(P<0.01)$. Also, the correlation between SBE and $\mathrm{NI}$ and AGPase, and AGPase and $\beta$-AMY were significant $(P<0.01)$. By contrast, no significant association between $\mathrm{NI}, \mathrm{Al}$ and $\beta$-AMY was observed. Al and SuSy showed no significant correlation with any other parameter at all.

Loading [MathJax]/jax/output/CommonHTML/jax.js 
Table 1

Pearson correlation between starch and sucrose content, enzyme activities and corm size

\begin{tabular}{|c|c|c|c|c|c|c|c|c|c|c|}
\hline & NI & Al & $\beta$-AMY & SPS & SuSy & AGPase & SBE & $\begin{array}{l}\text { Sucrose } \\
\text { content }\end{array}$ & $\begin{array}{l}\text { Starch } \\
\text { content }\end{array}$ & Diameter \\
\hline $\mathrm{NI}$ & 1 & & & & & & & & & \\
\hline Al & 0.428 & 1 & & & & & & & & \\
\hline$\beta$-AMY & 0.327 & 0.148 & 1 & & & & & & & \\
\hline SPS & $0.591^{*}$ & 0.372 & 0.478 & 1 & & & & & & \\
\hline SuSy & $0.622^{*}$ & $0.601^{*}$ & -0.047 & 0.439 & 1 & & & & & \\
\hline AGPase & 0.486 & 0.045 & $0.689^{\star *}$ & 0.497 & 0.100 & 1 & & & & \\
\hline SBE & $0.800^{\star \star}$ & 0.154 & $0.548^{*}$ & $0.651^{*}$ & 0.392 & $0.878^{\star \star}$ & 1 & . & & \\
\hline $\begin{array}{l}\text { Sucrose } \\
\text { content }\end{array}$ & $0.573^{*}$ & 0.000 & $0.559^{*}$ & $0.682^{\star \star}$ & 0.256 & $0.856^{\star \star}$ & $0.879^{\star \star}$ & 1 & & \\
\hline $\begin{array}{l}\text { Starch } \\
\text { content }\end{array}$ & $0.760^{\star \star}$ & 0.255 & $0.585^{*}$ & $0.662^{\star \star}$ & 0.458 & $0.857^{\star \star}$ & $0.957^{\star \star}$ & $0.921^{\star \star}$ & 1 & \\
\hline Diameter & $0.839^{\star \star}$ & 0.269 & 0.524 & $0.677^{\star \star}$ & 0.522 & $0.815^{\star \star}$ & $0.971^{\star *}$ & $0.889^{\star \star}$ & $0.980^{\star \star}$ & 1 \\
\hline
\end{tabular}

\section{Rna-seq And Transcriptome Assembly}

The sequencing statistics based on a mixed cDNA library of freesia corms at different developmental stages are summarized in Additional file 2: Tab. S2 and Additional file 3: Tab. S3. After data filtering, $82.54 \mathrm{~Gb}$ of clean reads were generated with Q30 $\geq 89.73$ and GC 48\% averagely. Totally 100,999 unigenes were generated after assembly with a mean length of $772 \mathrm{nt}$. The N50 was 1,507 nt and 21,476 unigenes were longer than 1,000 nt. On average, $63 \%$ of the genes were uniquely mapped.

\section{Unigene Annotation And Functional Classification}

In the current study, totally 44,405 unigenes were annotated with 41,175 annotated in Nr, 13,753 in COG, 25,142 in GO, 15,743 in KEGG, 23,015 in KOG, 24,441 in Swissport and 28,454 in Pfam (see Additional file 4: Tab. S4). Figure 3 shows that 13,753 sequences were classified into 24 COG categories, covering majority of life activities. Apart from "general function prediction only" (1,561 unigenes, $10.18 \%$ of annotated) and "translation, ribosomal structure and biogenesis" (1,460 unigenes, $9.52 \%)$, "carbohydrate transport and metabolism" term took the central position with 1432 unigenes annotated (9.34\%).

There are 25,142 unigenes annotated in GO database, which were classifies into three big functional categories, i.e., cellular component, molecular function and biological process (Fig. 4). The subcategories in the principle category of cellular component was most represented by "cell”, "membrane", "cell part" and "organelles". Within the principle category of molecular function, most genes participated in activities related to catalysis and binding. In the principle Loading [MathJax]/jax/output/CommonHTML/jax.js nostly involved in metabolic process, cellular process and single-organism 
process, indicative of occurrence of important metabolic and cellular activities in freesia corms. Notably, a considerable portion of genes were assigned to reproductive processes and development processes. All of these annotation information provides insights into investigation of potential genes involved in developmental processes of freesia corms.

\section{Distribution And Function Assignment Of Degs}

The expression levels of unigenes at different developmental stages were compared. As shown in Fig. 5, 3,427 DEGs in total from three pairwise comparisons were detected with the largest number distributed in the third pair (120 vs.190 d), where 2037 DEGs were identified with 1,551 down regulated and 486 upregulated. In comparison, 723 (290 up-regulated and 433 down-regulated) and 667 DEGs (520 up-regulated and 147-down regulated) were found in other two pairs (60 vs. $90 \mathrm{~d}$ and 90 vs. $120 \mathrm{~d}$, respectively). The contrasting results suggested that more complex biochemical activities may occur during the late developmental stages especially during the rapid swelling period.

GO and COG functional and KEGG enrichment pathway analysis was performed to gain further insights into DEGs. The major categories of GO terms in three pairwise comparisons (see Additional file 5: Fig. S1) were similar to that for the combined unigenes at four developmental stages as described in Fig. 4. Notably, DEGs between 120 and $190 \mathrm{~d}$ were enriched in "nutrient reservoir activity" pathway, which was void in other two comparisons. Comparatively, differences in COG enrichment profiles of DEGs in three comparisons were more pronounced (see Additional file 6: Fig. S2). Within the 60 vs. $90 \mathrm{~d}$ comparison, the term "posttranslational modification, protein turnover, chaperones" was at the top, followed by "carbohydrate transport and metabolism" and "general function prediction only". In the 90 vs. $120 \mathrm{~d}$ comparison, the top representative terms were "signal transduction mechanisms", "carbohydrate transport and metabolism" and "posttranslational modification, protein turnover, chaperones" sequentially. While within the 120 vs. $190 \mathrm{~d}$ comparison, "carbohydrate transport and metabolism" ranked the first, further suggesting that carbohydrate metabolic pathway might be most active during late stages of corm development. Pathway enrichment analysis revealed top 20 enriched pathways of DEGs in Additional file 7: Fig. S3. Generally, DEGs were, though in small quantity (< 5 or 10), significantly enriched in terms like riboflavin metabolism and diterpenoid biosynthesis in 60 vs. $90 \mathrm{~d}$ comparison, linoleic acid metabolism and monoterpenoid biosynthesis in 90 vs. $120 \mathrm{~d}$ comparison, and anthocyanin biosynthesis in 120 vs. 190 d comparison. Notably, DEGs enriched in starch and sucrose metabolism in the $120 \mathrm{vs.} 190 \mathrm{~d}$ comparison was quantitatively superior to other pathways, attenuating the greater activity of starch and sucrose metabolism in late developmental stages, in line with the results from GO and COG analysis.

\section{Verification Of Gene Expression By Qrt-pcr}

A keyword search in RNA-seq annotation generated a total of 39 putative genes encoding six carbohydrate-metabolizing enzymes investigated in this study, i.e., SPS, AGPase, SBE, $\beta$-AMY, SuSy and INV. The expression of these genes were displayed in Additional file 8: Tab. S5. To confirm the gene expression profiles of the enzymes from RNA-seq results, 6 out of the 39 genes (shown in bold and italic in Additional file 8: Tab. S5), including $\beta$-AMY1 (c62599.graph_c1), INV2 (c87855.graph_c0), SPS1 (c91035.graph_c1), SuSy (c93394.graph_c2), SBE4 (c78179.graph_c0) and AGPGase5 (c93923.graph_c0), were selected for qRT-PCR. Primer sequences of the genes for PCR were specified in Additional file 9: Tab. S6. The results of agarose gel electrophoresis evidenced that all 6 primer pairs amplified a single band (see Additional file 10: Fig. S4). Overall, the expression profiles of selected genes revealed by qRT-PCR matched that by RNAseq across four developmental stages (see Additional file 11: Fig. S5). Correlation analysis shows that the gene expression levels from qRT-PCR and RNA-seq are positively and significantly related $\left(r^{2}=0.7712, p<0.001\right)$ (Fig. 6). 
Among the 39 putative genes, averagely, most of them were differentially expressed across the corm developmental stages with homologues of AGPase, INV and SPS the most actively expressed (see Additional file 8: Tab. S5).

\section{Discussion}

\section{Carbohydrate metabolism regulates corm development}

Our previous work showed that the new corm formed $\sim 60$ day after mother corm embedment. Subsequently, corms expanded with a concomitant weight and size gain [19]. Different from tulipa [3] and lilies[7] with daughter bulbs appearing at the base of the parent bulbs, freesia showed an initial formation of a main corm at the joint of the parent corm and stem and later multiple tiny corms at the base or top of the main corm (see Additional file 1: Tab.S1). Therefore, at harvest, a main corm with several smaller corms were obtained. With respect to lilies, starch and sucrose content in mother bulbs was inversely correlated to that in the bulblets [7], suggesting that mother bulbs depleted gradually to support organogenesis in accompany with complex physiological changes. Carbohydrates in lily bulbs serve as primary nutrient and energy sources for substance synthesis,while soluble sugars, especially sucrose, are the main nutrients and energy source that can be easily translocated and used in the bulb [7].

Over the developmental stages of freesia corms, sucrose and starch exhibited an overall increasing trend, in agreement with the results reported on Lilium davidii var. unicolor [7] and Lycoris sprengeri bulbs [20]. At the formation stage, levels of sucrose and soluble sugars and starch were relatively low owing to the great nutritional need from mother corms for leaf development, and great photosynthate need for aerial biomass, allowing less carbohydrates allocated to new corms. After 90 days of planting, sucrose and starch accumulated simultaneously at the initial swelling stage. Similarly, the concomitant accumulation of sucrose and starch was observed in the initially expanded lily scales [21]. According to Fernie and Willmitzer [13], sucrose is crucial for potato tuber formation while starch is not necessary. Gao et al. [12] revealed that sucrose was responsible for induction and swelling of Lilium sargentiae bulblet associated to its physiological role and signaling function. The considerable level of sucrose observed in the current study may be a major trigger of new corm emergency and swelling. When the corm continued swelling, sucrose was unremittingly replenished by mother corms and photosynthetic apparatuses and the resultant increase of sucrose and soluble sugars in the corm laid a material basis for cell morphogenesis in the corm. Sucrose was postulated to be an critical factor for bulblet morphogenesis in lilies [7]. Till this time point, the new corm acted as a metabolic sink for energy input. Near the rapid swelling stage ( $120 \mathrm{~d}$ ), starch content accumulated sharply, likely because after cell morphogenesis, more photosynthates or sucrose were transformed to starch in corms for storage. At the end of rapid swelling stage ( $140 \mathrm{~d})$, freesia proceeded to full flowering phase when tiny corms emerged at the base of the main corm (see Additional file 1: Tab.S1); meanwhile, the main corm acted as a temporary source for tiny corm growth, the accumulation of starch hence slowed down. Since corms become mature, starch content continuously increased towards the end (190 d), suggesting that freesia corms acted as sink organs during maturation to reserve carbohydrate and energy, similar to Narcissus scales [22] and lily stolons [3]. Taken together, like lilies [16, 21, 23], starch and sucrose metabolism plays a center role in formation and development of freesia corms.

Sucrose levels are closely related to corresponding enzymes (Table 1). In the initial and rapid swelling period, although SPS activities surged, SuSy activities rose faster together with the enhancement of INV activities, which catalyzed the hydrolysis of sucrose, a compromise of sucrose synthesis by SPS, resulting in slow increase of sucrose content. At the corm maturation stage, SPS showed high activities while INV and SuSy activities declined, leading to sucrose uprise. SPS activities were paralleled with that of sucrose before $100 \mathrm{~d}$ but afterwards SPS activities reached a plateau in contrast to the steady increase of sucrose content. One possible explanation is that a high level of sucrose may inhibit the increase of SPS activities based on the proposition by Koch [24] that abundant sugar might adversely affect

Loading [MathJax]/jax/output/CommonHTML/jax.js Under this circumstance, sucrose content still rose continuously till the end 
of corm development but did not decline likely because of the constant replenishment of sucrose from mother bulb or aerial apparatuses. Correlation analysis indicated an insignificant correlation between SuSy and sucrose (Table 1), in contrast to the inverse correlation observed in tulip stolons [3], suggesting that decomposition effect of SuSy on sucrose was not pronounced herein. In the current study, activities of INV, especially Al, increased remarkably when corms entered the rapid swelling stage, in line with the findings that INV were very active in rapidly elongated meristems and rapid developed young organs or tissues where a concomitant slow accumulation of sucrose was observed [25]. Here, INV plays not only as a metabolic enzyme but possibly as a regulator in plant growth and organ establishment [26]. The subsequent decline of INV activities during corm maturation coincides with the statement in previous studies that INV activities tended to decline as related organs aged [27].

Starch metabolism is mainly regulated by APGase, SBE and $\beta$-AMY, and the relationship between them is significant (Table 1). APGase catalyzes the formation of a precursor (ADPG) required for starch synthesis [15]. Profiles of APGase activities, though more dynamic, generally paralleled with that of the starch. A notable drop of APGase activities occurred at $\sim 140 \mathrm{~d}$ when bulbs served as a temporary carbon source for the tiny corm formation, in accordance with drop of starch level in the main corm at the same time point. SBE is an important enzyme for amylopectin formation. Both starch content and SBE dramatically increased as the corm developed, a hint that amylopectin may be an important type of starch in the corm of freesia, which need to be further investigated. $\beta$-AMY cleaved starch into reducing sugars for corm growth [28]. $\beta$-AMY activities increased initially together with starch, while during bulb maturation it declined, when the nutrient requirement diminished and starch mainly served as a storage form of hydrocarbon, suggesting that $\beta$-AMY play an important role regulating and poising forms of hydrocarbons, in line with the results observed on lily bulbs [21]. Levels of starch result from a balance between consumption and production. Overall, dynamic changes of related enzymes implied that starch synthesis took the lead. Sucrose cleavage also contributes to the substrate for starch production [29]. Consequently, starch content presented an increasing trend across the new corm development and was the dominant form of carbohydrates in the present study.

\section{The Transcriptome And Freesia Corm Development}

So far, studies on freesia genome sequencing are rather lacking. Transcriptomic sequences vary in different plant organs. It is known that flowers of Freesia hybrida 'Pink Passion' and Freesia hybrida 'Jintong' yielded a total of 74,192 and 74,660 unigenes, among which 42,934 and 53,906 were annotated, respectively [30,31]. To our knowledge, this is the first report on freesia corms using RNA-sEq. In the present work, RNA-seq libraries for four developmental stages of freesia corms yielded 100,999 unigenes, larger than that in the previous studies. Of them, 44,405 unigenes (44\%) were annotated while about $56 \%$ were not, resulting from the limitation of genomic sequence information in freesia. On average, in the mixed library from four developmental stages, 9.3\% of annotated COG were connected to carbohydrate transport and metabolism (Fig. 3). Also, GO functional assignment analysis of unigenes directed "metabolic process" and "developmental process" important terms with gene enrichment, consistent with the involvement of genes related to carbohydrate transport and metabolism in corm development. Additionally, GO and COG functional classification of DEGs in three library pairs pinpointed the enrichment of DEGs between the rapid swelling (120 d) and maturation stages (190 d) in "carbohydrate transport and metabolism" or "nutrient reservoir activity" categories. DEGs annotated in "carbohydrate transport and metabolism" ranked the first quantitatively among all COG categories. Further, starch and sucrose metabolism was a significantly enriched KEGG term in the 120 vs. $190 \mathrm{~d}$ comparison but not in other two pairs. The 120 vs. 190 pair accounted for the largest portion of DEGs, and 76\% thereof were downregulated, while $78 \%$ of DEGs in the 90 vs. $120 \mathrm{~d}$ comparison were upregulated. Taken together, at transcriptional level, starch and sucrose metabolism was an essential metabolic pathway across the whole developmental process and was particularly active at the rapid swelling stage. As corms matured, activities of carbohydrate metabolism declined concurrent with wilting of

Loading [MathJax]/jax/output/CommonHTML/jax.js 
Transcriptome analysis of carbohydrate metabolism during bulb development, though relatively less addressed, was carried out in a number of bulbous or tuberous plants. Zhang et al. [14] performed transcriptome analysis of sucrose metabolism in onion (Allium cepa L.) bulbs and found 7\% of COG annotated unigenes were involved in carbohydrate transport and metabolism. And KEGG pathway enrichment analysis of DEGs revealed that "starch and sucrose metabolism" was the primary metabolism pathway in the integrated library from three developmental stages of onion bulbs and was most active at rapidly expanding stage, in line with the outcomes in the present work. Similarly, Li et al. [7] conducted transcriptome analysis of carbohydrate metabolisms in Lilium davidii var. unicolor bulblets and reported that $8 \%$ of annotated COG was associated to carbohydrate transport and metabolism. KEGG analysis of DEGs indicated that starch and sucrose metabolism was the principle pathway from three library pairs corresponding to three developmental stages of bulblets and was most active during bulblet formation stage. Elsewhere, the close association of carbohydrate metabolism with storage root development was also revealed on Lycoris sprengeri [20], sweet potato (Ipomoea batatas.) [32], and Sagittaria sagittifolia [33]. Altogether, these findings confirmed the important role of starch and sucrose metabolism in bulb formation and development of bulbous plants via transcriptome profiling method.

\section{Gene Expression During Corm Development}

A total of 6 genes out of the 39 putative genes encoding 6 key enzymes involved in starch and sucrose metabolism were selected for qRT-PCR to verify their expression profiles by RNA-sEq. The significant relationship between RNA-seq and qRT-PCR outcomes suggested that both methods are robust and can be complementary to each other for estimation of gene expression.

Expression of AGPase (AGPase 2, AGPase 5 and AGPase 6) and INV (INV1, INV 2 and INV 4) encoding genes (see Additional file 8: Tab. S5) peaked at $120 \mathrm{~d}$ (the rapid swelling stage) when starch and sucrose metabolic pathway was the most active and starch accumulated at the fastest rate, implying the critical roles of these genes in carbohydrate metabolism and starch accumulation. Comparatively, the relative expression level of SBE encoding gene (SBE4) was low before corm maturation but then increased by dozens of folds at $190 \mathrm{~d}$, seemingly indicating that SBE4 could be essential for starch accumulation during corm maturation. AGPase is a rate-limiting enzymes for starch biosynthesis since it catalyze the formation of ADPG [34], a precursor substrate for subsequent synthesis of amylose and amylopectin. SBEs cleave a-1,4 bonds and generate a-1,6-glucosidic bonds on C6 hydroxyls of an a-1,4 glucan which is essential for amylopectin formation [35]. The expression profile of SBE encoding gene possibly signified the variations in starch composition across corm development. Nevertheless, since amylose and amylopectin content was not quantified and amylose synthesis enzymes were not all determined in freesia corms, it is difficult to tell whether the ratio of amylose and amylopectin was altered during corm maturation. The heavy expression of AGPase and SBE at $120 \mathrm{~d}$ and $190 \mathrm{~d}$, respectively, likely suggested that the coordinated expression of them may regulate starch accumulation in the storage organ. INV genes are also significant regulators of starch accumulation since INV converts sucrose to glucose and fructose, which join the starch synthesis pathway. Elsewhere, AGPase encoding genes were reported to exhibited the highest expression at the middle and late-swelling stages of the Sagittaria sagittifolia bulb when starch and sucrose metabolism was most pronounced while INV and SBE genes did not [33]. Additionally, Zhang et al. [32] found that AGPase, soluble starch synthase, granule-bound starch synthase and SuSy encoding genes mostly peaked in sweet potatoes at $95 \mathrm{~d}$ after planting when starch accumulation peaked. Apparently, the genes mainly responsible for starch accumulation varies with plant type. Here, we did not align gene expression profiles to that of sucrose accumulation because sucrose accumulation was not merely a result of enzymatic action in the developing corm but may also be contributed, to a large extent, by long-distance translocation inward.

By comparison, we found that the gene expression patterns did not always follow that of corresponding enzyme Loading [MathJax]/jax/output/CommonHTML/jax.js gesting that, at the transcriptional level, the expression of these genes may 
not play a decisive role regulating variations of enzyme activities during corm development. One possible explanation is that enzyme synthesis is comprehensively regulated by multiple genes of unequal contribution, which may oscillate between a positive and a negative direction in the transcriptional regulatory network. Similar inconsistences between gene expression levels and activities of starch metabolic enzymes were also observed in Lycoris sprengeri bulbs [20] and Tulipa edulis stolons [3]. In view of the limitation in gene annotation and the number of key enzymes related to starch and sucrose metabolism investigated in the current study, more important homologous genes of adequate key enzymes remain to be determined and their roles to be mined. Regardless, the obtained results provide valuable resources for further in-depth investigations of the molecular mechanisms involved in starch and sucrose metabolism in freesia corm development.

\section{Conclusions}

In conclusion, in the current study, we investigated the physiology of starch and sucrose metabolism in freesia corms at four developmental stages. Obviously, corm development was paralleled with dynamics of starch and sucrose which were the comprehensive effects of carbohydrate-metabolizing enzymes. Transcriptome profiling provide further insights into molecular mechanisms underlying the metabolic process. Comparison of gene expression profiles with that of enzyme activities and carbohydrate content, comprehensively, it is suggested that AGPase, INV and SBE and may be critical for starch accumulation in the corm. To our knowledge, this is the first transcriptome analysis of starch and sucrose metabolism during corm formation and development in freesia. The results will shed light on elucidation of molecular mechanisms in corm formation and development and benefit for future improvements in molecular breeding of freesia.

\section{Methods}

\section{Sample collecting}

Freesia hybrida 'SN Huangjin' was planted in late October, 2016 in a farm on Minhang campus, Shanghai Jiao Tong University, China. Plants were first sampled at the 60th day after mother corm embedment and the following samples were collected at an interval of 10 days, resulting in 14 sampling events (i.e., 60, 70, 80, 90, 100, 110, 120, 130, 140, 150, $160,170,180$ and $190 \mathrm{~d}$ after planting). Our previous work detailed the biological characteristics of the plant and divided the developmental process of the new corm into four stages, i.e., the formation (60-90 d), initial swelling (90$120 \mathrm{~d})$, rapid swelling (120-140 d) and maturation (140-190 d) stages [19]. Pictures of corms at differential developmental stages were displayed in Additional file 1: Tab. S1. The plants were thoroughly rinsed with water to remove dirt and the newly developed corms were collected for analysis. At each sampling event, 10 healthy corms of similar size were pulverized, homogenized, divided into multiple portions prior to flash freezing with liquid nitrogen for storage $\left(-80^{\circ} \mathrm{C}\right)$, waiting for analysis. Three biological replicates for each treatment and three technical replicates within each biological sample were performed throughout the study.

\section{Determination Of Carbohydrate Content And Related Enzyme Activities}

Contents of the carbohydrates and activities of corresponding enzymes were determined in corms collected at 14 sampling events. Sucrose was determined according to Deng et al. [36]. Soluble sugars and starch were detected based on anthrone method and colorimetric assay of iodine, respectively [3]. Enzymes were extracted as described by Keller et al. [37]. Among them SPS and SuSy activities were evaluated using procedures by Wei et al. [38] and Datir and Joshi

Loading [MathJax]/jax/output/CommonHTML/jax.js

Page 10/21 
[39], Al and NI activities by Crusciol et al. [40] and SBE activities by Fu et al. [41]. Beta-AMY activities were determined by spectrophotometry.

\section{Rna Extraction, Cdna Library Construction, Sequencing And Assembly}

Corms sampled at 60, 90, 120 and 190 d, representing four developmental stages of new corms (e.g, formation, initial swelling, rapid swelling and maturation stages) respectively, were selected for transcriptomic analysis. RNA was extracted using RNApre Pure Plant Kit (Tiangen Biotech, Beijing, China) following the manufacture's protocol. An equal portion of RNA extracted from four developmental stages (sampled at 60, 90, 120 and $190 \mathrm{~d}$ ) each and their combination were subject to library construction, sequencing and assembly. Quality of RNA was detected using a NanoDrop 2000 spectrophotometer (Thermo Scientific, Waltham, MA, USA) and gel electrophoresis (1\%). Clear bands and appropriate A260/A280 (1.9-2.1) and A260/A230 (>2.0) values obtained in this study suggested that the RNA quality was good enough for library construction. RNA-seq was performed by BioMarker Biotechnology Corporation (Beijing, China) based on Illumina (CA, USA). Raw data were filtered to generate clean reads which were de novo assembled by Trinity to obtain unigenes [42].

\section{Gene Functional Annotation}

Unigenes were aligned, by BLASTx (E-value $\leq 1 \mathrm{e}-5$ ), to NCBI Non-redundant Protein (Nr), Gene Ontology (GO), Clusters of Orthologous Groups of proteins (COG), Kyoto Encyclopedia of Genes and Genomes (KEGG), euKaryotic Clusters of Orthologous Groups (KOG), Swissprot and evolutionary genealogy of genes: Non-supervised Orthologous Groups (eggnog) databases for functional annotation. Unigenes were searched against the Pfam database by HMMER with an E-value $\leq 1 \mathrm{e}-10$. Functional annotation by GO terms was carried out by Blast2Go [43]. The KEGG pathway annotation was performed online using KEGG Automatic Annotation Server (https://www.genome.jp/tools/kaas/).

\section{Analysis Of Differentially Expressed Genes (degs)}

The transcript abundance of each unigene was calculated and normalized to fragments per kilobase of transcript per million mapped reads (FPKM) value [44]. DEGs between different developmental stages were screened using DESeq software [45]. Obtained $P$ values was corrected using Benjamini-Hochbergs method to account for multiple tests using the false discovery rate (FDR). Genes with FDR $<0.01$ and absolute expression fold change (log2 scaled) $\geq 4$ were deemed to be significantly differentially expressed. Identified DEGs were then subject to GO database for functional analysis and to KEGG for identification of metabolic pathways.

Quantitative real-time polymerase chain reaction (qRT-PCR) analysis of genes of key enzymes involved in sucrose and starch metabolism

Putative genes of key enzymes involved in sucrose and starch metabolism were retrieved from KEGG results and their expression was verified by qRT-PCR. RNA was extracted and purified. The first-strand cDNA was synthesized using Prime Script ${ }^{\mathrm{TM}}$ RT reagent kit with gDNA Eraser (TaKaRa, Dalian, China) and then used as template for PCR. Primers, designed using Primer 5.0 software, were provide by Qingxi Biology (Shanghai, China). Real time qRT-PCR was conducted in a reaction volume of $20 \mu \mathrm{L}$ containing $10 \mu \mathrm{l}$ of SYBR, $7.4 \mu \mathrm{L}$ of double-distilled $\mathrm{H}_{2} \mathrm{O}, 0.8 \mu \mathrm{L}$ of each primer and $1 \mu \mathrm{L}$ of cDNA mix. Relative gene expression levels were acquired using the $2^{-\Delta \Delta C T}$ method after normalizing to actin 


\section{Abbreviations}

SuSy

sucrose synthase; SPS:sucrose phosphate synthase; INV:invertase; NI:neutral invertase; Al:acid invertase;

APGase:adenosine diphosphoglucose pyrophosphorylase; ADPG:adenosine diphosphate glucose; SBE:starch branching enzyme; AMY:amylase; RNA-seq:RNA sequencing; Nr:NCBI Non-redundant Protein; GO:Gene Ontology; COG:Clusters of Orthologous Groups of proteins; KEGG:Kyoto Encyclopedia of Genes and Genomes; KOG:euKaryotic Clusters of Orthologous Groups; DEG:differentially expressed gene; FPKM:fragments per kilobase of transcript per million mapped reads; FDR:false discovery rate; qRT-PCR:quantitative real-time polymerase chain reaction; FW:fresh weight

\section{Declarations}

\section{Ethics approval and consent to participate}

Not applicable

\section{Consent for publication}

Not applicable

\section{Availability of data and materials}

The datasets generated and/or analysed during the current study are not publicly available due to the consideration that the manuscript has not been published but are available from the corresponding author on reasonable request.

\section{Competing interests}

The authors declare that they have no competing interests.

\section{Funding}

This work was financially supported by the Agricultural Commission of Shanghai, China, under Grant Hu Nong Ke Gong (2014) no. 1-2.

\section{Author's contributions}

Dongqin Tang conceived and designed the experiment. Suqin Ding performed the experiment. Zi Yan assisted Suqin Ding in sample collection. Li Ma analyzed the data and prepared the manuscript. All authors read and approved the final version of the manuscript.

\section{Acknowledgements}

Not applicable

\section{References}


1. Wang L. Freesia. In: Anderson NO, editor. Flower breeding and genetics: Issues, challenges and opportunities for the 21st century. Dordrecht: Springer Netherlands; 2006. pp. 665-93.

2. Miao YY, Zhu ZB, Guo QS, Yang XH, Liu L, Sun Y, et al. Dynamic changes in carbohydrate metabolism and endogenous hormones during Tulipa edulis stolon development into a new bulb. J Plant Biol. 2016;59(2):121-32.

3. Miao YY, Guo QS, Zhu ZB, Yang XH, Wang CL, Sun Y, et al. Dynamic changes in starch metabolism and endogenous hormones during stolon formation in tulipa edulis. J Am Soc Hort Sci. 2016;141(3):211-21.

4. Vishnevetsky J, Zamski E, Ziv M. Carbohydrate metabolism in Nerine sarniensis bulbs developing in liquid culture. Physiol Plant. 2000;108(4):361-9.

5. Xia Y, Zheng H, Huang C, editors. Studies on the bulb development and its physiological mechanisms in Lilium oriental hybrids. IX International Symposium on Flower Bulbs 673; 2004.

6. Jing YL, Liu F, Zhou YW. Study on carbohydrate metabolism changes of enlarging lily bulblet in cold region. Bei Fang Yuan Yi. 2010:74-6.

7. Li X, Wang C, Cheng J, Zhang J, da Silva JAT, Liu X, et al. Transcriptome analysis of carbohydrate metabolism during bulblet formation and development in Lilium davidii var. unicolor. BMC Plant Biol. 2014;14(1):358.

8. Ohyama T, Ikarashi T, Matsubara T, Baba A. Behavior of carbohydrates in mother and daughter bulbs of tulips (Tulipa gesneriana). Soil Sci Plant Nutr. 1988;34(3):405-15.

9. Wu S-s, Wu J-d, Jiao X-h, Zhang Q-x, Lv Y-m. The dynamics of changes in starch and lipid droplets and sub-cellular localization of $\beta$-amylase during the growth of lily bulbs. J Integr Agric. 2012;11(4):585-92.

10. Martínez MT, Vieitez AM, Corredoira E. Improved secondary embryo production in Quercus alba and Q. rubra by activated charcoal, silver thiosulphate and sucrose: influence of embryogenic explant used for subculture. Plant Cell Tiss Org Cult. 2015;121(3):531-46.

11. Rook F, Gerrits N, Kortstee A, van Kampen M, Borrias M, Weisbeek P, et al. Sucrose-specific signalling represses translation of the Arabidopsis ATB2 bZIP transcription factor gene. Plant J. 1998;15(2):253-63.

12. Gao S, Zhu Y, Zhou L, Fu X, Lei T, Chen Q, et al. Sucrose signaling function on the formation and swelling of bulblets of Lilium sargentiae EH Wilson. Plant Cell Tiss Org Cult. 2018;135(1):143-53.

13. Fernie AR, Willmitzer L. Molecular and biochemical triggers of potato tuber development. Plant Physiol. 2001;127(4):1459-65.

14. Zhang CS, Zhang HW, Zhan ZX, Liu BJ, Chen ZT, Liang Y. Transcriptome analysis of sucrose metabolism during bulb swelling and development in onion (Allium cepa L.). Front Plant Sci. 2016;7.

15. Rouhier $\mathrm{H}$, Usuda $\mathrm{H}$. Spatial and temporal distribution of sucrose synthase in the radish hypocotyl in relation to thickening growth. Plant Cell Physiol. 2001;42(6):583-93.

16. Wu S, Peng D, Li W, Wang J. Carbohydrate metabolism and activity variation of related enzymes during the exchanging role of bulb source and sink of oriental hybrid lily'Sorbonne'. Journal of Beijing Forestry University. 2013;35(6):96-102.

17. Zhe YZHXX, Mingfang Y. Metabolism and related enzymes activities of sucrose and starch in the stages of bulb formation of Gladiolus hybridus [J]. Sci Silvae Sin. 2008;44(8):47-51.

18. Onda Y, Mochida K. Exploring genetic diversity in plants using high-throughput sequencing techniques. Curr Genomics. 2016;17(4):358-67.

19. Ding S, Yan Z, Li X, Tang D. Study on the change of endogenous hormones during bulb development in Freesia hybrida. J Agric Sci Technol. 2019;21(9):51-7.

20. Chang L, Xiao YM, She LF, Xia YP. Analysis of gene expression and enzyme activities related to starch metabolism in Lvcoris sprenaeri bulbs of different sizes. Sci Hortic. 2013;161:118-24.

Loading [MathJax]/jax/output/CommonHTML/jax.js

Page $13 / 21$ 
21. Sun H, Li T, Li Y. Changes of carbohydrate and amylase in lily bulb during bulb development. Zhi Wu Yan Jiu. 2005;25(1):59-63.

22. Ruamrungsri S, Ruamrungsri S, Ikarashi T, Ohyama T. Carbohydrate metabolism in Narcissus. J Horticult Sci Biotechnol. 1999;74(3):395-400.

23. Sun H-m, He L, Wang W-w, Jia Z-k, Li T-I. Mechanism of starch-sucrose metabolism regulated by IBA as well as GA_3 during scale cutting propagation in Lilium. Zhongguo Nong Ye Ke Xue. 2011;44(4):798-806.

24. Koch KE. Carbohydrate-modulated gene expression in plants. Annu Rev Plant Physiol Plant Mol Biol. 1996;47(1):509-40.

25. Arai M, Mori H, Imaseki H. Roles of sucrose-metabolizing enzymes in growth of seedlings. Purification of acid invertase from growing hypocotyls of mung bean seedlings. Plant Cell Physiol. 1991;32(8):1291-8.

26. Roitsch T, Gonzalez MC. Function and regulation of plant invertases: sweet sensations. Trends Plant Sci. 2004;9(12):606-13.

27. Zhang M-F, Li Z-L. Sucrose-metabolizing enzymes in higher plants. Plant Physiol Commun. 2002;38(3):289-95.

28. Sawai J, Nakai T, Hashimoto A, Shimizu M. A comparison of the hydrolysis of sweet potato starch with betaamylase and infrared radiation allows prediction of reducing sugar production. Int J Food Sci Technol. 2004;39(9):967-74.

29. Bánfalvi Z, Molnar A, Molnar G, Lakatos L, Szabo L. Starch synthesis, and tuber storage protein genes are differently expressed in Solanum tuberosum and in Solanum brevidens. FEBS Lett. 1996;383(3):159-64.

30. Huang ML, Fan RH, Ye XX, Lin RY, Luo YH, Fang NY, et al. The transcriptome of flower development provides insight into floral scent formation in Freesia hybrida. Plant Growth Regul. 2018;86(1):93-104.

31. Tang D-Q, Sun Y, Li X, Yan Z, Shi Y-M. De novo sequencing of the Freesia hybrida petal transcriptome to discover putative anthocyanin biosynthetic genes and develop EST-SSR markers. Acta Physiol Plant. 2018;40:168.

32. Zhang K, Wu ZD, Tang DB, Luo K, Lu HX, Liu YY, et al. Comparative transcriptome analysis reveals critical function of sucrose metabolism related-enzymes in starch accumulation in the storage root of sweet potato. Front Plant Sci. 2017;8:914.

33. Gao MP, Zhang SW, Luo C, He XH, Wei SL, Jiang W, et al. Transcriptome analysis of starch and sucrose metabolism across bulb development in Sagittaria sagittifolia. Gene. 2018;649:99-112.

34. Geigenberger P. Regulation of starch biosynthesis in response to a fluctuating environment. Plant Physiol. 2011;155(4):1566-77.

35. Tetlow IJ, Emes MJ. A review of starch-branching enzymes and their role in amylopectin biosynthesis. IUBMB Life. 2014;66(8):546-58.

36. Deng CJ, Chang-Qing LI, Zhu XQ, Guo XP. Determination of total sugars and residual sugars from aureobiasidium pullulans broth by anthrone-sulfuric acid colorimetry method. Food Drug. 2006;8(11):54-6.

37. Keller F, Ludlow M. Carbohydrate metabolism in drought-stressed leaves of pigeonpea (Cajanus cajan). J Exp Bot. 1993;44(8):1351-9.

38. Wei W, Qin GY, Wang LL, Yan W, Ge JZ, Huang DQ, et al. Changes of augar content and sucrose metabolizing enzyme activities during fleshy taproot development in radish (Raphanus sativus L.). Yuan Yi Xue Bao. 2007;34(5):1313-6.

39. Datir S, Joshi S. The contribution of sucrose metabolism enzymes to sucrose accumulation in sugarcane (Saccharumofficinarum L.) genotypes. Indian J Plant Physiol. 2016;21(1):76-82.

40. Crusciol CAC, Leite GHP, de Siqueira GF, Silva MD. Response of application of growth inhibitors on sugarcane productivitv and sucrose accumulation in the middle of cropping season in Brazil. Sugar Tech. 2017;19(2):155-64.

Loading [MathJax]/jax/output/CommonHTML/jax.js

Page $14 / 21$ 
41. Fu GF, Li H, Tao LX, Zhang XF, Wang DY. Effects of shading at grain-filling stage on the growth and Q enzyme activity of rice grain. Chinese J Ecol. 2009;28(3):438-44.

42. Grabherr MG, Haas BJ, Yassour M, Levin JZ, Thompson DA, Amit I, et al. Full-length transcriptome assembly from RNA-Seq data without a reference genome. Nat Biotechnol. 2011;29(7):644-U130.

43. Gotz S, Garcia-Gomez JM, Terol J, Williams TD, Nagaraj SH, Nueda MJ, et al. High-throughput functional annotation and data mining with the Blast2GO suite. Nucleic Acids Res. 2008;36(10):3420-35.

44. Trapnell C, Williams BA, Pertea G, Mortazavi A, Kwan G, van Baren MJ, et al. Transcript assembly and quantification by RNA-Seq reveals unannotated transcripts and isoform switching during cell differentiation. Nat Biotechnol.

2010;28(5):511-U174.

45. Anders S, Huber W. Differential expression analysis for sequence count data. Genome Biol. 2010;11(10).

\section{Figures}
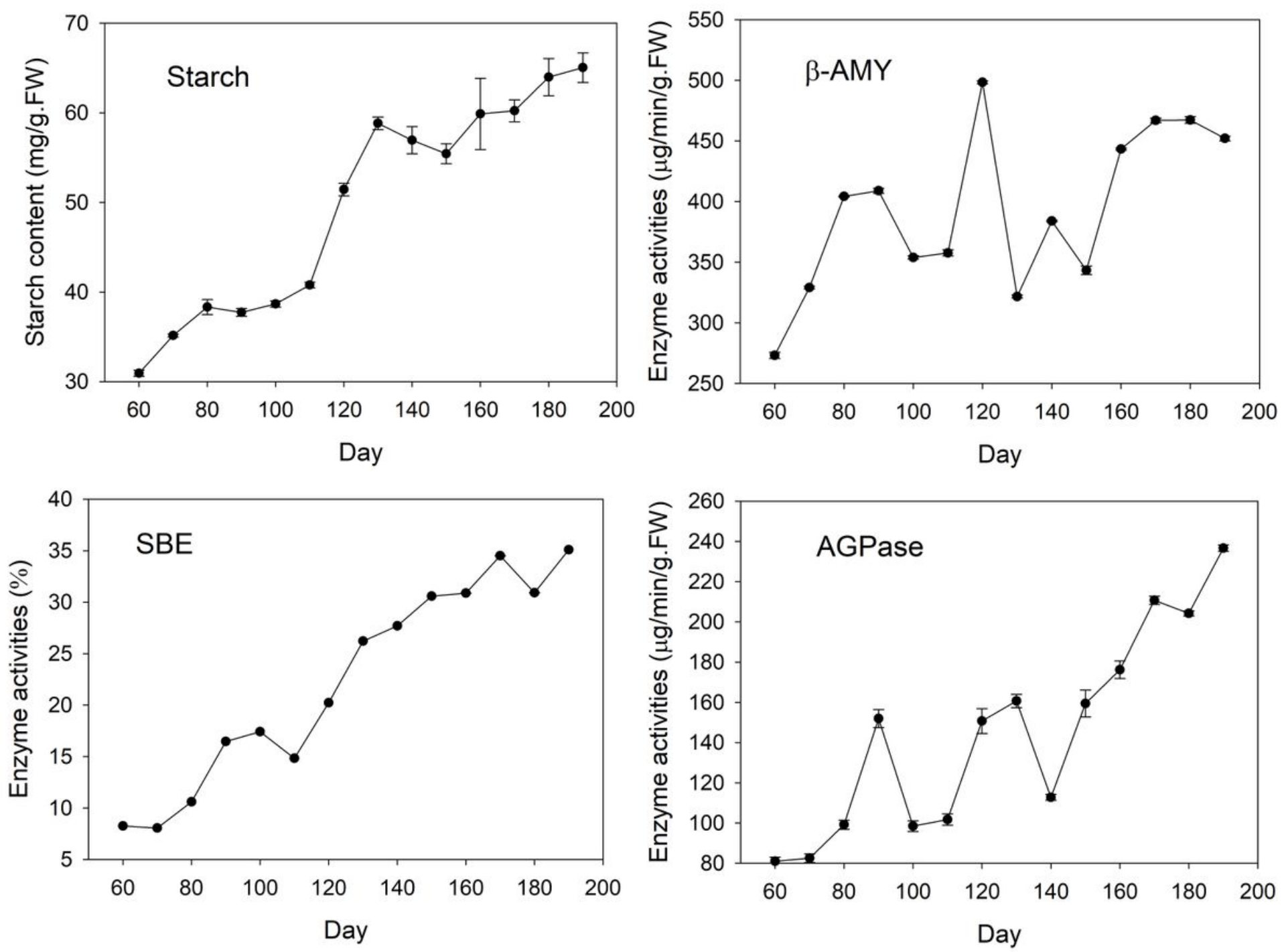

Figure 1 
Changes of starch content and activities of corresponding enzymes during freesia corm development. AMY: amylase; SBE: starch branching enzyme; AGPase: adenosine diphosphoglucose pyrophosphorylase; FW: fresh weight
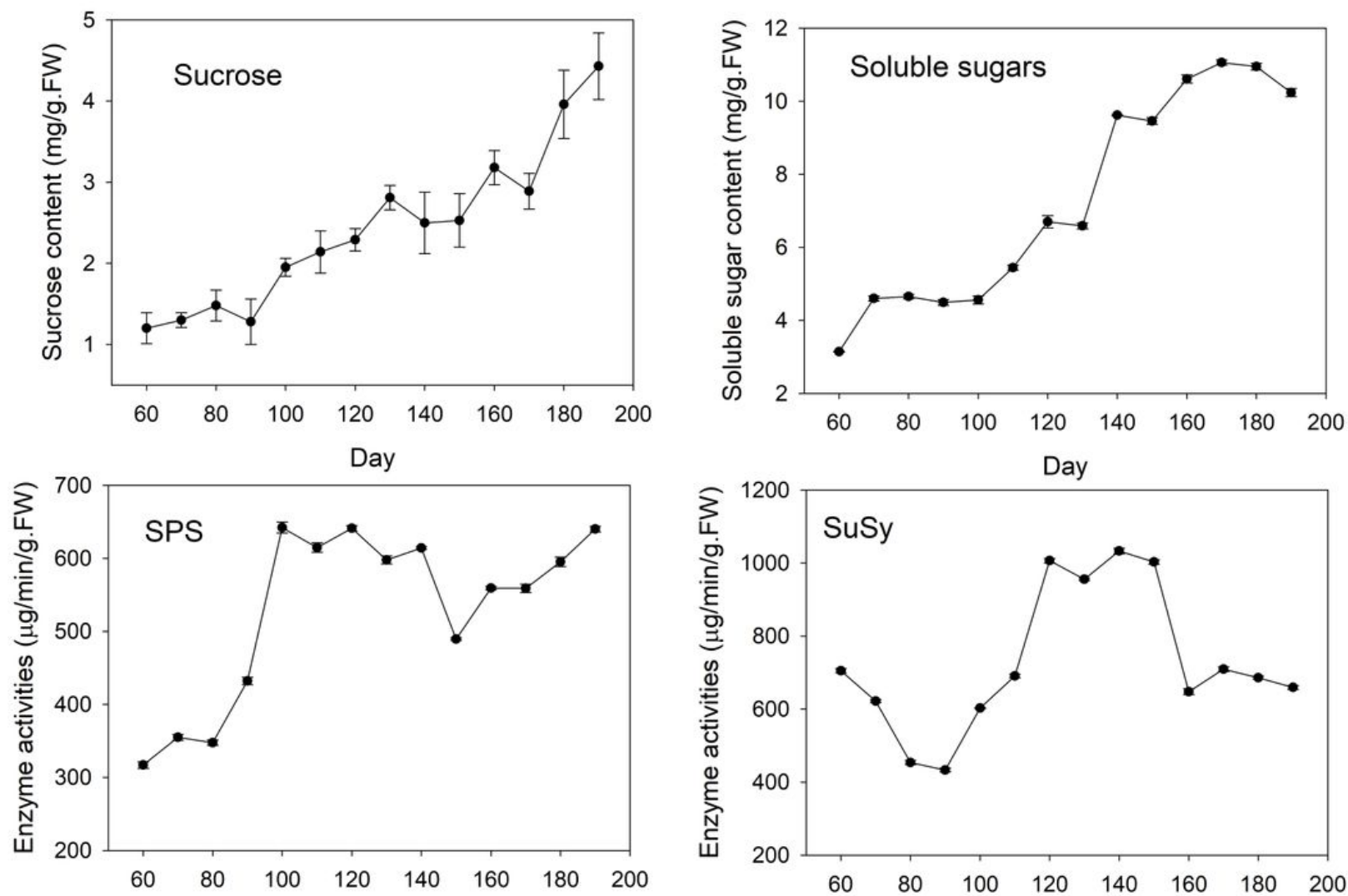

Day
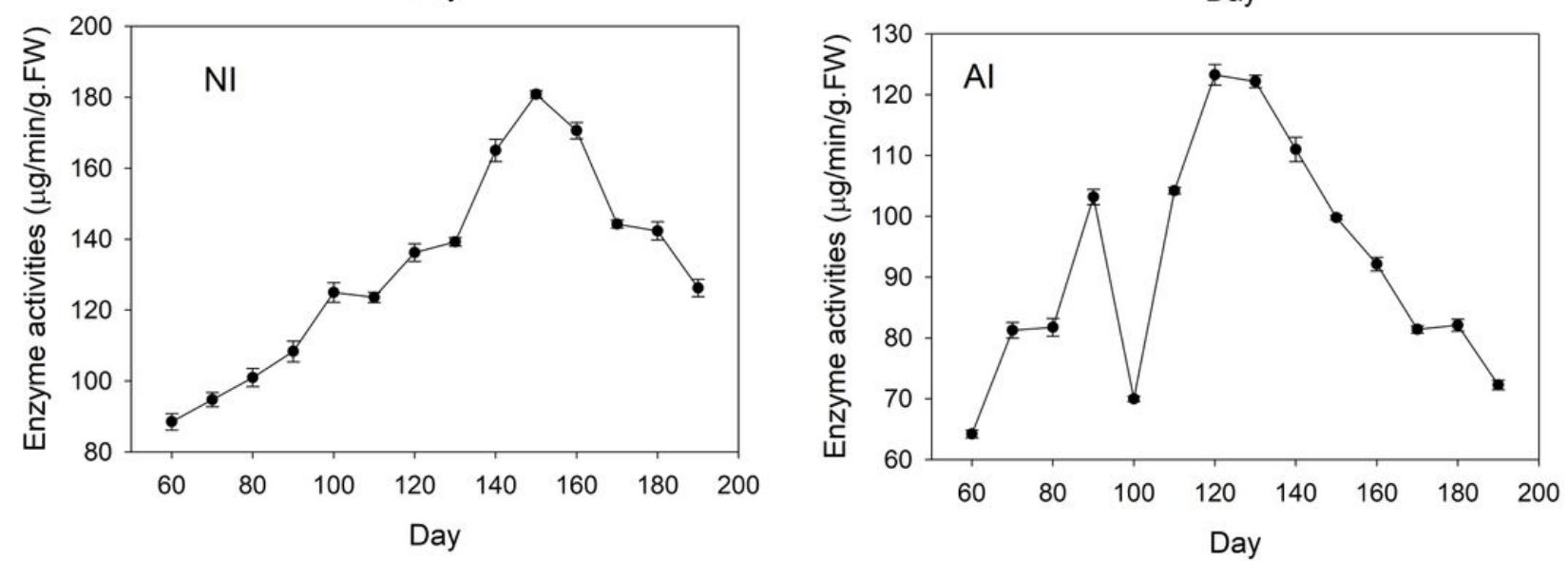

Figure 2

Changes of sugar content and activities of corresponding enzymes during freesia corm development. SPS: sucrose phosphate synthase; SuSy: sucrose synthase, Nl: neutral invertase; Al: acid invertase; FW: fresh weight 


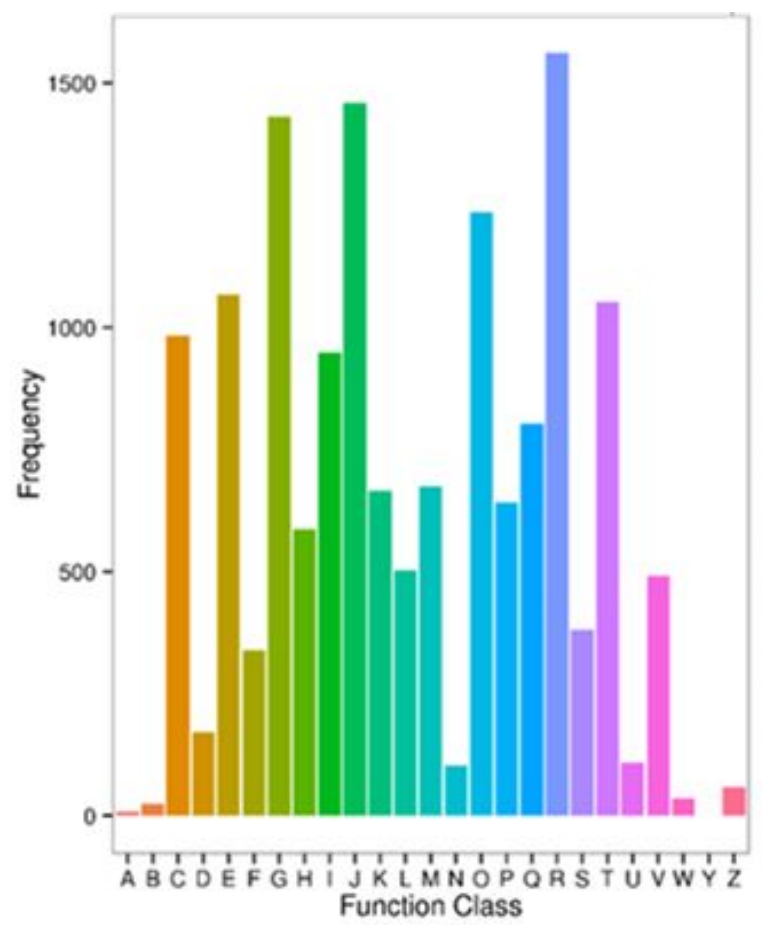

A: RNA processing and modification [9-0.06\%]

B: Chromatin structure and dynamics $[24-0.16 \%]$

C: Energy production and conversion [984-6,42\%]

D: Cell cycle control, cell division, chromosome partitioning [171 1.12\%]

E: Amino acid transport and metabolism [1067-6.96\%]

F: Nucleoside transport and metabolism [339-2.21\%]

G: Carbohydrate transpont and metabolsm [1432-9.34\%]

$\mathrm{H}$ : Coenzyme transport and metabolism [587 -3.83\%]

I: Lipid transport and metabolism [948-6.18\%]

J: Translation, ribosomal structure and biogenesis [1460-9.52\%]

K: Transcription [665 -4.34\%]

L: Replication, recombination and repair [503 -3.28\%]

M: Cell wallmembrane'envelope biogenesis $[674 \sim 4.4 \%]$

N: Cell mosifity [103-0.67\%]

O: Posttranslational modification, protein turnover, chaperones [1236-8.06\%]

P: Inorganic ion transpont and metabolism [641 -4.18\%]

Q: Seconday metabolites biosynthesis, transport and catabolism [802 $-5.23 \%$ ]

R: General function prediction only $[1561-10.18 \%]$

S: Function urknown [380-2.48\%]

T: Signal transduction mechanisms [1052 -6.86\%]

$\mathrm{U:} \mathrm{Intracellular} \mathrm{trafficking.} \mathrm{secretion,} \mathrm{and} \mathrm{vesicular} \mathrm{transport} \mathrm{[109-0.71 \% ]}$

V: Defense mechanisms [491 -3.2\%]

W: Extracellular structures [36 - 0.23\%]

Y: Nuclear structure [0-0\%]

Z: Cytoskeleton [59-0.38\%]

Figure 3

COG function classifications of unigenes in freesia corms from the mixed library of four developmental stages 


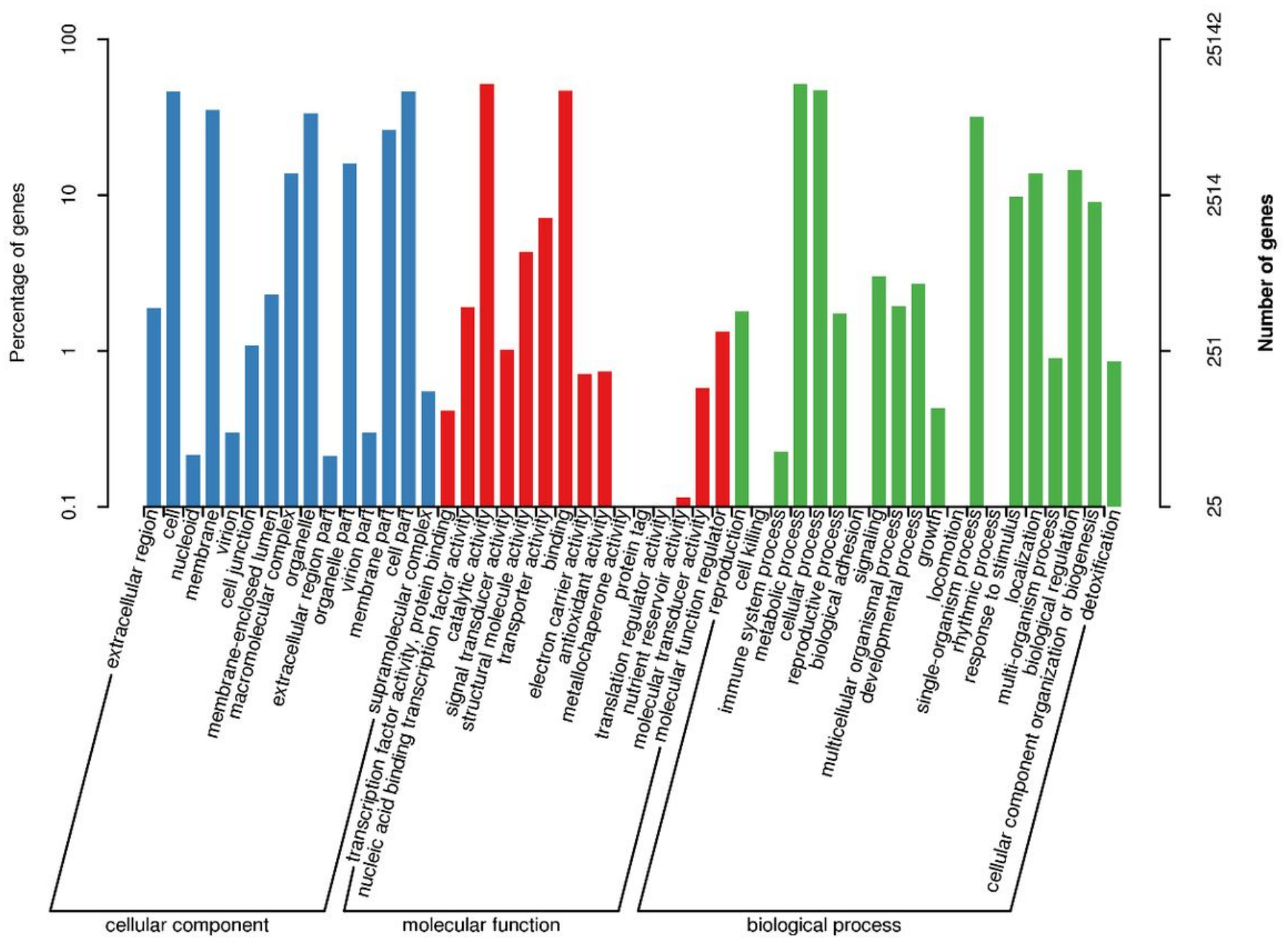

Figure 4

GO function classification of unigenes in freesia corms from the integrated library of four developmental stages. The yaxis represents the subgroups in the three $\mathrm{GO}$ annotation, while the $\mathrm{x}$-axis represents the percentage of genes matching the specific category within each of the primary categories 


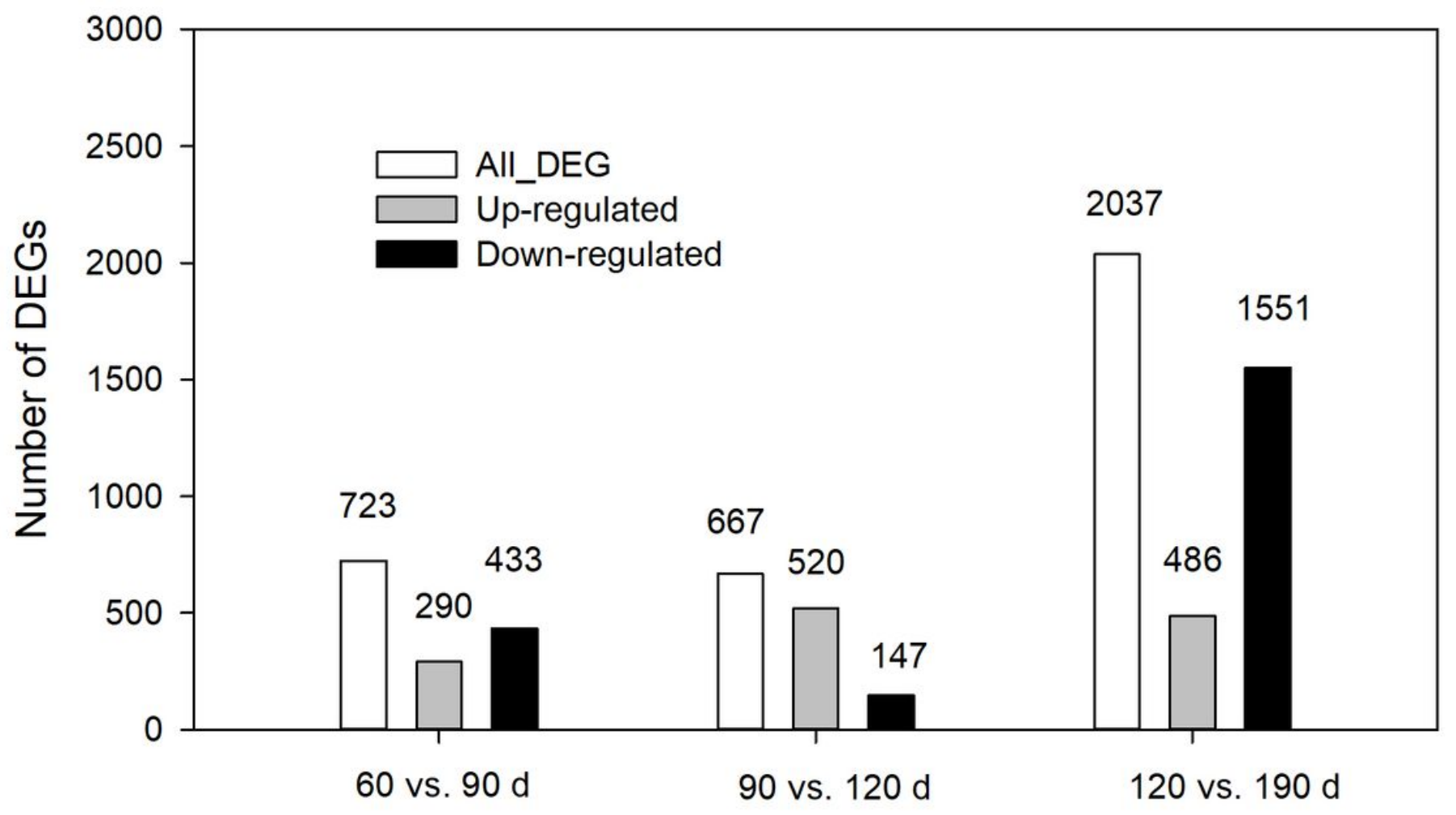

Figure 5

Statistics of differentially expressed genes (DEGs) at different development stages of corms 


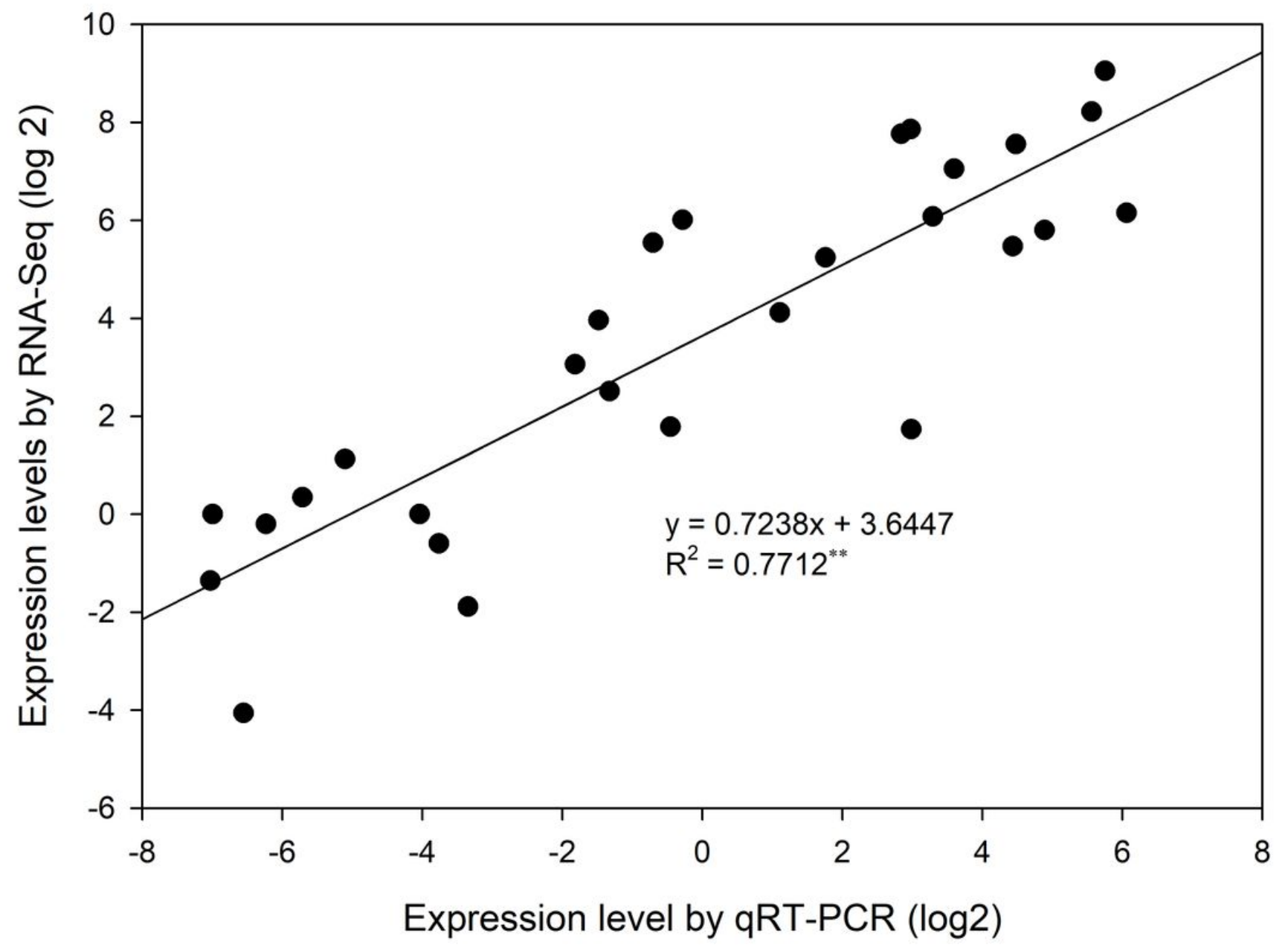

Figure 6

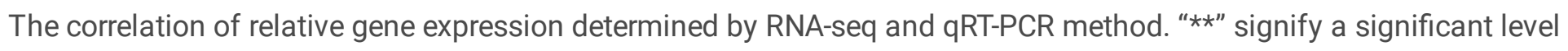
of 0.01 .

\section{Supplementary Files}

This is a list of supplementary files associated with this preprint. Click to download.

- Additionalfile2.docx

- Additionalfile5.docx

- Additionalfile6.docx

- Additionalfile9.docx

- Additionalfile11.docx

- Additionalfile10.docx

- Additionalfile7.docx

- Additionalfile1.docx 
- Additionalfile3.docx

- Additionalfile8.docx 